\title{
Minor Prime Factorization for $n$-D Polynomial Matrices over Arbitrary Coefficient Field
}

\author{
Jinwang Liu, Dongmei Li $@$, and Licui Zheng \\ School of Mathematics and Computing Sciences, Hunan University of Science and Technology, Xiangtan, Hunan, China 411201 \\ Correspondence should be addressed to Dongmei Li; dmli@hnust.edu.cn
}

Received 11 January 2018; Revised 4 September 2018; Accepted 10 September 2018; Published 8 October 2018

Academic Editor: Eulalia Martínez

Copyright (C) 2018 Jinwang Liu et al. This is an open access article distributed under the Creative Commons Attribution License, which permits unrestricted use, distribution, and reproduction in any medium, provided the original work is properly cited.

\begin{abstract}
In this paper, we investigate two classes of multivariate $(n-D)$ polynomial matrices whose coefficient field is arbitrary and the greatest common divisor of maximal order minors satisfy certain condition. Two tractable criterions are presented for the existence of minor prime factorization, which can be realized by programming and complexity computations. On the theory and application, we shall obtain some new and interesting results, giving some constructive computational methods for carrying out the minor prime factorization.
\end{abstract}

\section{Introduction}

In the polynomial approach, pioneered by Rosenbrock [1], matrices over $R[s], s \equiv d / d t$ are used to represent linear systems of ordinary differential equations. For more general linear functional systems, e.g., partial differential systems or delay-differential systems, the resulting system matrices are multivariate. The problem of multivariate $(n-\mathrm{D})$ polynomial matrix has attracted much attention over the past decades because of its diverse applications in $n$-D systems, network theory, Wiener-Hopf equations, controls, and signal processing $([2-8])$; its factorizations have attracted much attention over the past decades because of their wide applications ([9-25]). Lin-Bose conjecture is one of the important problems of $n$-D polynomial matrix factorizations, which has been proved in [15-20]. It gave a sufficient condition for a matrix to have zero prime factorization or minor prime factorization. After that, Lin et al. proposed a tractable criterion for the existence of minor prime factorizations for a class of $n$-D polynomial matrices whose greatest common divisor of maximal order minors is $z_{1}-f\left(z_{2}, \cdots, z_{n}\right)$. They have presented a constructive method for carrying out the minor prime factorization when it exists [14], where the coefficient field is the complex number field. In this paper, we investigate the $n$-D polynomial matrices whose coefficient field is arbitrary and minor prime factorizations for two classes of $n$-D polynomial matrices whose the greatest common divisor of maximal order minors is $z_{1}-f\left(z_{2}, \cdots, z_{n}\right)$ or $\prod_{k=1}^{p}\left(z_{1}-z_{1 k}\right)$. In this case, because the coefficient field is an arbitrary field, the many previous results (lemmas) cannot be used directly. By giving some new key lemmas, two tractable criterions for the existence of minor prime factorization are obtained. These criterions can be judged by using "programming of reduced Gröbner basis." Furthermore, we give constructive computational methods for carrying out the minor prime factorization.

\section{Notations and Preliminaries}

For an arbitrary field $K$, let $K\left[z_{1}, z_{2}, \cdots, z_{n}\right]$ denote the set of polynomial ring in $n$ variables $z_{1}, z_{2}, \ldots, z_{n}$ with coefficients in $K$ and $\bar{K}$ denote an algebraic closed field of $K$. For the convenience, $K\left[z_{1}, z_{2}, \cdots, z_{n}\right]$ is denoted simply by $K[z]$; when $K=C$ is the complex number field, $C\left[z_{1}, z_{2}, \cdots, z_{n}\right]$ is denoted simply by $C[z]$. Let $K^{l \times m}[z]$ denote the set of $l \times m$ matrices with entries in $K[z]$ and $0_{r \times t}$ denote the $r \times t$ zero matrices. Throughout the paper, the argument $(z)$ is omitted whenever its omission does not cause confusion.

Definition 2.1 (see [21]). Let $F(z) \in K^{l \times m}(z)$ be of normal rank $r$ and $a_{1}(z), a_{2}(z), \ldots, a_{\beta}(z)$ denote all the $r \times r$ minors 
of the matrix $F(z)$. Extracting the greatest common divisor (g.c.d.), denoted by $d(F)$ or $d$, of $a_{1}(z), a_{2}(z), \ldots, a_{\beta}(z)$ gives

$$
a_{i}(z)=d b_{i}(z), \quad i=1,2, \ldots, \beta .
$$

Then, $b_{1}(z), b_{2}(z), \ldots, b_{\beta}(z)$ are called the reduced minors of $F(z)$.

Definition 2.2. Let $F(z) \in K^{l \times m}[z]$ be of normal full rank, then $F(z)$ is said to be zero left prime (zero right prime) if the $l \times l(m \times m)$ minors of $F(z)$ generate the unit ideal $K[z]$, which is simplified as that $F(z)$ is ZLP (ZRP).

First, the following two lemmas and a well-known result (Theorem 1 in [14]) are required.

Lemma 2.1. Let $g(z) \in K[z]$ and $f(z) \in K\left[z_{2}, \cdots, z_{n}\right]$. Supposing that $g\left(f, z_{2}, \cdots, z_{n}\right)=0$, then $z_{1}-f\left(z_{2}, \cdots, z_{n}\right)$ is a divisor of $g(z)$.

The proof is simple and omitted.

Lemma 2.2. Let $f_{1}(z), f_{2}(z), \ldots, f_{s}(z) \in K[z]$ be polynomials, then they have no common zeros in $\bar{K}^{n}$ (are zero coprime) if and only if there are $u_{1}(z), u_{2}(z), \ldots, u_{s}(z) \in K[z]$ such that

$$
u_{1}(z) f_{1}(z)+u_{2}(z) f_{2}(z)+\cdots+u_{s}(z) f_{s}(z)=1,
$$

i.e., $\left(f_{1}(z), f_{2}(z), \cdots, f_{s}(z)\right)$ is a ZLP row vector in $K^{n}[z]$, or $f_{1}(z), \ldots, f_{s}(z)$ generate the unit ideal $K[z]$.

Proof 1. Necessity. It is clear that $f_{i}(z) \in \bar{K}[z], i=1,2, \ldots, s$. Let $I_{K}$ and $I_{\bar{K}}$ denote the ideal generated by $f_{1}(z), f_{2}(z), \ldots$, $f_{s}(z)$ in $K[z]$ and in $\bar{K}[z]$, respectively. From the assumption and Hilbert Zero Theorem, $I_{\bar{K}}=\bar{K}[z]$. Then the unit element 1 (in $\bar{K}$ ) may be represented by a Gröbner basis $G$ of $I_{\bar{K}}$, i.e., 1 may be reduced to 0 by $G$. According to computational methods of $G$, we have that $G \subset K[z]$, then 1 can be represented by $G$ in $K[z]$, i.e., there are $u_{1}(z), u_{2}(z), \ldots, u_{s}(z)$ $\in K[z]$ such that

$$
u_{1}(z) f_{1}(z)+u_{2}(z) f_{2}(z)+\cdots+u_{s}(z) f_{s}(z)=1
$$

Sufficiency. We assume that $f_{1}(z), f_{2}(z), \ldots, f_{s}(z)$ have common zeros in $\bar{K}^{n}$ and $z_{0}=\left(z_{11}, z_{21}, \cdots, z_{n 1}\right)$ is one of them. From (2), we have

$$
u_{1}\left(z_{0}\right) f_{1}\left(z_{0}\right)+u_{2}\left(z_{0}\right) f_{2}\left(z_{0}\right)+\cdots+u_{s}\left(z_{0}\right) f_{s}\left(z_{0}\right)=0 \neq 1
$$

This is a contradiction. So $f_{1}(z), f_{2}(z), \ldots, f_{s}(z)$ have no common zeros in $\bar{K}^{n}$.

Theorem 2.1 (see [14]). Let $F(z) \in C^{m \times l}[z], d(F)=z_{1}-$ $f\left(z_{2}, \cdots, z_{n}\right)$. Then a necessary and sufficient condition for $F$ $(z)$ to admit a minor prime factorization $F(z)=F_{0}(z) G_{0}(z)$ with $F_{0}(z) \in K^{m \times l}[z], G_{0}(z) \in K^{l \times l}[z]$, and det $G_{0}(z)=d(F)$ is that there exists a full rank $(l-1) \times l$ submatrix $F_{1}\left(z_{2}, \cdots, z_{n}\right)$ $\triangleq F_{1}\left(f\left(z_{2}, \cdots, z_{n}\right), z_{2}, \cdots, z_{n}\right)$ of $F\left(f\left(z_{2}, \cdots, z_{n}\right), z_{2}, \cdots, z_{n}\right)$ such that the reduced minors of $F_{1}\left(z_{2}, \cdots, z_{n}\right)$ are zero coprime.

\section{Main Results}

For the complex number field $C$ and an $n$-D polynomial matrix $F\left(z_{1}, z_{2}, \cdots, z_{n}\right) \in C[z]$, from Lemma 2.2, we see that the reduced minors of $F\left(z_{1}, z_{2}, \cdots, z_{n}\right)$ are zero coprime if and only if the reduced minors of $F\left(z_{1}, z_{2}, \cdots, z_{n}\right)$ generate the unit ideal $C[z]$. In the following, we investigate the case in which $F\left(z_{1}, z_{2}, \cdots, z_{n}\right) \in K[z]$, where $K$ is an arbitrary field (may be a finite field); we shall extend the range of applicability of Theorem 2.1 and present our main results. First, several key lemmas are presented.

Lemma 3.1. Let $Q \in K^{(l-1) \times l}[z]$ be of normal full rank, if the reduced minors of $Q$ generate $K[z]$, then there is a ZLP matrix $w \in K^{l \times 1}[z]$ such that $Q \cdot w=0_{(l-1) \times 1}$.

Proof 2. Since the reduced minors of $Q$ generate the unit ideal $K[z]$, by Lin-Bose Theorem (Theorem 3.3 in [18]), $Q$ can be factorized as $Q=Q_{1} B_{1}$ such that $Q_{1} \in R^{(l-1) \times(l-1)}$ is of normal full rank, and $B_{1} \in K^{(l-1) \times l}$ is ZRP. By Quillen-Suslin Theorem [26], there is a unimodular $U_{1} \in K^{l \times l}[z]$ such that $B_{1}$ is its first $l-1$ rows, i.e., $U_{1}=\left(B_{1}^{T} V_{1}^{T}\right)^{T}$, where $V_{1} \in K^{1 \times l}$. Let $U_{1}^{-1}=\left(\begin{array}{ll}V_{2} & w\end{array}\right)$, where $V_{2} \in R^{l \times(l-1)}, w \in R^{l \times 1}$, then $B_{1}$ $w=0_{(l-1) \times 1}$. Obviously, $w$ is ZLP. We have that $Q \cdot w=Q_{1}$. $B_{1} \cdot w=Q_{1} \cdot 0_{(l-1) \times l}=0_{(l-1) \times 1}$.

Lemma 3.2. Let $F(z) \in K^{m \times l}[z](l \leq m)$ and $d(F)=\prod_{s=1}^{q}$ $\left(z_{1}-f_{s}\left(z_{2}, \cdots, z_{n}\right)\right)$, where $f_{i}-f_{j} \neq 0$ for $i \neq j$. Then rank $F$ $\left(f_{p}\left(z_{2}, \cdots, z_{n}\right), z_{2}, \cdots, z_{n}\right)$ is $l-1$, where $p=1,2, \ldots, q$.

Proof 3. Let $F(z)=\left(f_{i j}\right)_{m \times l}$, for $p \in\{1,2, \cdots, q\}$, we may write $f_{i j}$ as

$$
f_{i j}=q_{i j}\left(z_{1}-f_{p}\left(z_{2}, \cdots, z_{n}\right)\right)+b_{i j}
$$

where $q_{i j} \in K[z], \quad b_{i j} \in K\left[z_{2}, \cdots, z_{n}\right], i=1,2, \ldots, m, j=1,2$, $\ldots, l$.

Setting $A \triangleq\left(a_{i j}\right)_{m \times l} \triangleq\left(q_{i j}\left(z_{1}-f_{p}\left(z_{2}, \cdots, z_{n}\right)\right)\right)_{m \times l}, \quad B \triangleq$ $\left(b_{i j}\right)_{m \times l}$, we have that $F=A+B$. Since $d(F)=\prod_{s=1}^{q}\left(z_{1}-f_{s}\right.$ $\left.\left(z_{2}, \cdots, z_{n}\right)\right)$, then there is an $l \times l$ minor $\Delta$ of $F(z)$ such that $z_{1}-f_{p}\left(z_{2}, \cdots, z_{n}\right)$ is a simple divisor of $\Delta$. Let $F\left(j_{1}, j_{2}, \cdots, j_{l}\right)$ denote an $l \times l$ submatrix consisting of the $j_{1}, j_{2}, \ldots, j_{l}$ rows of $F$ such that $z_{1}-f_{p}\left(z_{2}, \cdots, z_{n}\right)$ is a simple divisor of $\operatorname{det} F\left(j_{1}, j_{2}, \cdots, j_{l}\right)$. Then

$$
F\left(j_{1}, j_{2}, \cdots, j_{l}\right)=\left(\begin{array}{cccc}
a_{j_{1} 1}+b_{j_{1} 1} & a_{j_{1} 2}+b_{j_{1} 2} & \cdots & a_{j_{1} l}+b_{j_{1} l} \\
a_{j_{2} 1}+b_{j_{2} 1} & a_{j_{2} 2}+b_{j_{2} 2} & \cdots & a_{j_{2} l}+b_{j_{2} l} \\
\cdots & \cdots & \cdots & \cdots \\
a_{j_{l} 1}+b_{j_{l} 1} & a_{j_{l} 2}+b_{j_{l} 2} & \cdots & a_{j_{l} l}+b_{j_{l} l}
\end{array}\right) .
$$


Since $\left(z_{1}-f_{p}\left(z_{2}, \cdots, z_{n}\right)\right) \mid a_{i j}$, where $i=1,2, \ldots, m, j=1$, $2, \ldots, l, b_{i j}$ is independent of $z_{1}$, and $z_{1}-f_{p}\left(z_{2}, \cdots, z_{n}\right)$ is a simple divisor of $\operatorname{det} F\left(j_{1}, j_{2}, \cdots, j_{l}\right)$, by the properties of determinant, some $k(1 \leq k \leq l)$ exists such that

$$
\left|\begin{array}{ccccccc}
b_{j_{1} 1} & \cdots & b_{j_{1} k-1} & a_{j_{1} k} & b_{j_{1} k+1} & \cdots & b_{j_{1} l} \\
b_{j_{2} 1} & \cdots & b_{j_{2} k-1} & a_{j_{2} k} & b_{j_{2} k+1} & \cdots & b_{j_{2} l} \\
\cdots & \cdots & \cdots & \cdots & \cdots & \cdots & \cdots \\
b_{j_{l} 1} & \cdots & b_{j_{l} k-1} & a_{j_{l} k} & b_{j_{l} k+1} & \cdots & b_{j_{l} l}
\end{array}\right| \neq 0 .
$$

From (7) and Laplace Theorem, there is a permutation $i_{1} i_{2} \cdots i_{l-1}$ of $j_{1} j_{2} \cdots j_{l}$ such that

$$
\Delta_{k} \triangleq\left|\begin{array}{cccccc}
b_{i_{1} 1} & \cdots & b_{i_{1} k-1} & b_{i_{1} k+1} & \cdots & b_{i_{1} l} \\
b_{i_{2} 1} & \cdots & b_{i_{2} k-1} & b_{i_{2} k+1} & \cdots & b_{i_{2} l} l \\
\cdots & \cdots & \cdots & \cdots & \cdots & \cdots \\
b_{i_{l-1} 1} & \cdots & b_{i_{l_{1}} k-1} & b_{i_{l-1} k+1} & \cdots & b_{i_{l-1} 1}
\end{array}\right| \neq 0 .
$$

It is obvious that $F\left(f_{p}\left(z_{2}, \cdots, z_{n}\right), z_{2}, \cdots, z_{n}\right)=B$, then $\Delta_{k}$ is an $(l-1) \times(l-1)$ minor of $F\left(f_{p}\left(z_{2}, \cdots, z_{n}\right), z_{2}, \cdots, z_{n}\right)$. Since $d\left(F\left(f_{p}\left(z_{2}, \cdots, z_{n}\right), z_{2}, \cdots, z_{n}\right)\right)=0$, combined with (8), we have that rank $F\left(f_{p}\left(z_{2}, \cdots, z_{n}\right), z_{2}, \cdots, z_{n}\right)$ is $l-1$.

Lemma 3.3. Let $F(z) \in K^{l \times l}[z]$ and $\operatorname{det}(F)=\prod_{s=1}^{q}\left(z_{1}-f_{s}\right.$ $\left.\left(z_{2}, \cdots, z_{n}\right)\right)$, where $0 \neq f_{i}-f_{j} \in K$ for $i \neq j$. Then rank $F\left(f_{p}\left(z_{2}, \cdots, z_{n}\right), z_{2}, \cdots, z_{n}\right)=l-1$ for every $\left(z_{2}, \cdots, z_{n}\right) \in$ $\bar{K}^{n-1}$, and rank $F\left(f_{p}\left(z_{2}, \cdots, z_{n}\right), z_{2}, \cdots, z_{n}\right)=l-1$; furthermore, the $(l-1) \times(l-1)$ minors of $F\left(f_{p}\left(z_{2}, \cdots, z_{n}\right), z_{2}\right.$, $\left.\cdots, z_{n}\right)$ generate the unit ideal $K[z]$, where $p=1,2, \ldots, q$.

Proof 4. For arbitrary but fixed $z_{2}=z_{20}, \ldots, z_{n}=z_{n 0}$, where $\left(z_{20}, \cdots, z_{n 0}\right) \in \bar{K}^{n-1}$, let $z_{1 p}=f_{p}\left(z_{20}, \cdots, z_{n 0}\right)$, then $z_{11}, z_{12}$, $\ldots, z_{1 q}$ are distinguished. Assuming that $F\left(z_{1}, z_{20}, \cdots, z_{n 0}\right)$ $=\left(f_{i j}\right)_{l \times l}$, for $p \in\{1,2, \cdots, q\}$, we may write $f_{i j}$ as

$$
f_{i j}=q_{i j}\left(z_{1}-z_{1 p}\right)+b_{i j}
$$

where $q_{i j} \in \bar{K}\left[z_{1}\right], b_{i j} \in \bar{K}, i, j=1,2, \ldots, l$.

Setting $A \triangleq\left(a_{i j}\right)_{l \times l} \triangleq\left(q_{i j}\left(z_{1}-z_{1 p}\right)\right)_{l \times l}, B \triangleq\left(b_{i j}\right)_{l \times l}$, then we have that $F\left(z_{1}, z_{20}, \cdots, z_{n 0}\right)=A+B$,

$\operatorname{det} F\left(z_{1}, z_{20}, \cdots, z_{n 0}\right)=\left(\begin{array}{cccc}a_{11}+b_{11} & a_{12}+b_{12} & \cdots & a_{1 l}+b_{1 l} \\ a_{21}+b_{21} & a_{22}+b_{22} & \cdots & a_{2 l}+b_{2 l} \\ \cdots & \cdots & \cdots & \cdots \\ a_{l 1}+b_{l 1} & a_{l 2}+b_{l 2} & \cdots & a_{l l}+b_{l l}\end{array}\right)$.

Since det $F\left(z_{1}, z_{20}, \cdots, z_{n 0}\right)=\prod_{s=1}^{q}\left(z-z_{1 s}\right),\left(z_{1}-z_{1 p}\right) \mid a_{i j}$, where $i, j=1,2, \ldots, l$, and $b_{i j} \in \bar{K}, z_{1}-z_{1 p}$ is a simple divisor of det $F\left(z_{1}, z_{20}, \cdots, z_{n 0}\right)$, by the properties of determinant, there is some $k(1 \leq k \leq l)$ such that

$$
\left|\begin{array}{ccccccc}
b_{11} & \cdots & b_{1 k-1} & a_{1 k} & b_{1 k+1} & \cdots & b_{1 l} \\
b_{21} & \cdots & b_{2 k-1} & a_{2 k} & b_{2 k+1} & \cdots & b_{2 l} \\
\cdots & \cdots & \cdots & \cdots & \cdots & \cdots & \cdots \\
b_{l 1} & \cdots & b_{l k-1} & a_{l k} & b_{l k+1} & \cdots & b_{l l}
\end{array}\right| \neq 0 .
$$

From (11) and Laplace Theorem, there is a permutation $i_{1} i_{2} \cdots i_{l-1}$ of $12 \cdots l$ such that

$$
\Delta_{k} \triangleq\left|\begin{array}{cccccc}
b_{i_{1} 1} & \cdots & b_{i_{1} k-1} & b_{i_{1} k+1} & \cdots & b_{i_{1} l} \\
b_{i_{2} 1} & \cdots & b_{i_{2} k-1} & b_{i_{2} k+1} & \cdots & b_{i_{2} l} l \\
\cdots & \cdots & \cdots & \cdots & \cdots & \cdots \\
b_{i_{l_{1} 1} 1} & \cdots & b_{i_{l-1} k-1} & b_{i_{l-1} k+1} & \cdots & b_{i_{l-1} 1}
\end{array}\right| \neq 0 .
$$

It is obvious that $F\left(f_{p}\left(z_{20}, \cdots, z_{n 0}\right), z_{20}, \cdots, z_{n 0}\right)=B$, then $\Delta_{k}$ is an $(l-1) \times(l-1)$ minor of $F\left(f_{p}\left(z_{20}, \cdots, z_{n 0}\right), z_{20}, \cdots\right.$, $\left.z_{n 0}\right)$. Since det $\left(F\left(f_{p}\left(z_{20}, \cdots, z_{n 0}\right), z_{20}, \cdots, z_{n 0}\right)\right)=0$, combined with (12), we have that $\operatorname{rank} F\left(f_{p}\left(z_{2}, \cdots, z_{n}\right), z_{2}, \cdots, z_{n}\right)$ is $l-1$ for every $\left(z_{2}, \cdots, z_{n}\right) \in \bar{K}^{n-1}$, i.e., the $(l-1) \times(l-1)$ minor of $F\left(f_{p}\left(z_{2}, \cdots, z_{n}\right), z_{2}, \cdots, z_{n}\right)$ have no common zeros in $\bar{K}^{n-1}$. Noting that $\operatorname{det} F\left(f_{p}\left(z_{2}, \cdots, z_{n}\right), z_{2}, \cdots, z_{n}\right)=0$, then $\operatorname{rank} F\left(f_{p}\left(z_{2}, \cdots, z_{n}\right), z_{2}, \cdots, z_{n}\right)=l-1$. Furthermore, by Lemma 2.2 , we obtain that the $(l-1) \times(l-1)$ minors of $F\left(f_{p}\left(z_{2}, \cdots, z_{n}\right), z_{2}, \cdots, z_{n}\right)$ generate the unit ideal $K[z]$.

Corollary 3.1. Let $F(z) \in K^{l \times l}[z]$ with $\operatorname{det} F(z)=\prod_{k=1}^{q}\left(z_{1}-\right.$ $\left.z_{1 k}\right)$, where $z_{11}, z_{12}, \ldots, z_{1 q} \in K$ are distinguished. Then rank $F\left(z_{1 k}, z_{2}, \cdots, z_{n}\right)=l$, the $(l-1) \times(l-1)$ minors of $F\left(z_{1 k}, z_{2}\right.$, $\left.\cdots, z_{n}\right)$ generate the unit ideal $K[z]$, where $k=1,2, \ldots, q$.

Lemma 3.4. Let $G(z) \in K^{l \times l}[z]$, $\operatorname{det} G(z)=\prod_{s=1}^{q}\left(z_{1}-f_{s}\left(z_{2}\right.\right.$, $\left.\cdots, z_{n}\right)$ ), where $0 \neq f_{i}-f_{j} \in K$ for $i \neq j$. Then, for $p \in\{1,2$, $\cdots, q\}$,

$$
G\left(f_{p}\left(z_{2}, \cdots, z_{n}\right), z_{2}, \cdots, z_{n}\right)=\bar{G}(z) \cdot \operatorname{diag}\{0,1, \cdots, 1\} \cdot V_{p},
$$

where $\bar{G}(z) \in K^{l \times l}\left[z_{2}, \cdots, z_{n}\right], \quad V_{p} \in K^{l \times l}\left[z_{2}, \cdots, z_{n}\right] \quad$ is an invertible matrix.

Proof 5. Since $\operatorname{det} G(z)=\prod_{s=1}^{q}\left(z_{1}-f_{s}\left(z_{2}, \cdots, z_{n}\right)\right)$, for $p \in$ $\{1,2, \cdots, q\}$, by Lemma 3.3 , rank $G\left(f_{p}\left(z_{2}, \cdots, z_{n}\right), z_{2}, \cdots\right.$, $\left.z_{n}\right)=l-1$ and the $(l-1) \times(l-1)$ minors of $G\left(f_{p}\left(z_{2}, \cdots\right.\right.$, $\left.\left.z_{n}\right), z_{2}, \cdots, z_{n}\right)$ generate $K[z]$. By Lemma 3.1 , there is a ZLP vector $w_{p} \in K^{l \times 1}\left[z_{2}, \cdots, z_{n}\right]$ such that $G \cdot F\left(f_{p}\left(z_{2}, \cdots, z_{n}\right)\right.$, $\left.z_{2}, \cdots, z_{n}\right) \cdot w_{p}\left(z_{2}, \cdots, z_{n}\right)=(0, \cdots, 0)^{T}$. According to Quillen- 
Suslin Theorem [26], a unimodular matrix $U_{1} \in K^{l \times l}\left[z_{2}, \cdots\right.$, $\left.z_{n}\right]$ can be constructed such that $w_{p}\left(z_{2}, \cdots, z_{n}\right)$ is its first column, then

$$
G\left(f_{p}\left(z_{2}, \cdots, z_{n}\right), z_{2}, \cdots, z_{n}\right) \cdot U_{1}=\bar{G}(z) \cdot \operatorname{diag}\{0,1, \cdots, 1\},
$$

where $\bar{G}(z) \in K^{l \times l}\left[z_{2}, \cdots, z_{n}\right]$. Setting $V_{p}=U_{1}^{-1}$, then the desired results are obtained.

Lemma 3.5. Let $F(z) \in K^{(l-1) \times l}[z]$, if $F(z)$ is of normal full rank and $F(z)$ has the following factorization:

$$
F(z)=A(z) \cdot \operatorname{diag}\{0,1, \cdots, 1\} \cdot V(z)
$$

where $A(z) \in K^{(l-1) \times l}[z], \quad V(z) \in K^{l \times l}[z]$ is a unimodular matrix. Then the reduced minors of $F(z)$ generate the unit ideal $K[z]$.

Proof 6. We partition $A(z), V(z)$ as the following form, respectively,

$$
\begin{aligned}
& A(z)=\left(\begin{array}{ll}
A_{1}(z) & A_{2}(z)
\end{array}\right), \\
& V(z)=\left(\begin{array}{ll}
V_{1}^{T}(z) & V_{2}^{T}(z)
\end{array}\right)^{T},
\end{aligned}
$$

where $A_{1}(z) \in \mathrm{K}^{(l-1) \times 1}[z], A_{2}(z) \in K^{(l-1) \times(l-1)}[z], V_{1}(z) \in K^{1 \times l}$ $[z], V_{2}(z) \in K^{(l-1) \times l}[z]$. Then $V_{2}(z)$ is ZLP, i.e., the reduced minors of $V_{2}(z)$ generate the unit ideal $K[z]$, and

$$
F(z)=\left(\begin{array}{ll}
0 & A_{2}(z)
\end{array}\right) \cdot\left(\begin{array}{ll}
V_{1}^{T}(z) \quad V_{2}^{T}(z)
\end{array}\right)^{T}=A_{2}(z) \cdot V_{2}(z)
$$

So the reduced minors of $F(z)$ are the same as that of $V_{2}(z)$, which generate the unit ideal $K[z]$.

Theorem 3.1. Let $K$ be an arbitrary field, $F(z) \in K^{m \times l}[z]$ be of normal full rank $(l \leq m), d(F)=z_{1}-f\left(z_{2}, \cdots, z_{n}\right) \triangleq d(z)$. Then a necessary and sufficient condition for $F(z)$ to admit a minor prime factorization $F(z)=F_{0}(z) G_{0}(z)$ with $F_{0}(z) \epsilon$ $K^{m \times l}[z], G_{0}(z) \in K^{l \times l}[z]$, and $\operatorname{det} G_{0}(z)=d(z)$ is that there exists a normal full rank $(l-1) \times l$ submatrix $F_{1}\left(z_{2}, \cdots, z_{n}\right)$ $\triangleq F_{1}\left(f\left(z_{2}, \cdots, z_{n}\right), z_{2}, \cdots, z_{n}\right)$ of $F\left(f\left(z_{2}, \cdots, z_{n}\right), z_{2}, \cdots, z_{n}\right)$ such that the reduced minors of $F_{1}\left(z_{2}, \cdots, z_{n}\right)$ generate the unit ideal $K[z]$.

Proof 7. Necessity. Supposing that $F(z)$ admits a minor prime factorization

$$
F(z)=F_{0}(z) G_{0}(z)
$$

with $F_{0}(z) \in K^{m \times l}[z], G_{0}(z) \in K^{l \times l}[z]$, and $\operatorname{det} G_{0}(z)=z_{1}-$ $f\left(z_{2}, \cdots, z_{n}\right)=d(z)$. From Lemma 3.4, $G_{0}\left(f\left(z_{2}, \cdots, z_{n}\right), z_{2}\right.$, $\left.\cdots, z_{n}\right)=\overline{G_{0}}(z) \cdot \operatorname{diag}\{0,1, \cdots, 1\} \cdot V$, where $V \in K\left[z_{2}, \cdots, z_{n}\right]$ is unimodular. Combined with (18), we have

$$
\begin{aligned}
F\left(f\left(z_{2}, \cdots, z_{n}\right), z_{2}, \cdots, z_{n}\right)= & F_{0}\left(f\left(z_{2}, \cdots, z_{n}\right), z_{2}, \cdots, z_{n}\right) \\
& \cdot G_{0}\left(f\left(z_{2}, \cdots, z_{n}\right), z_{2}, \cdots, z_{n}\right) \\
= & F_{0}\left(f\left(z_{2}, \cdots, z_{n}\right), z_{2}, \cdots, z_{n}\right) \\
& \cdot \overline{G_{0}}(z) \cdot \operatorname{diag}(0,1, \cdots, 1) \cdot V .
\end{aligned}
$$

By Lemma 3.2, we see that the normal rank of $F\left(f\left(z_{2}, \cdots, z_{n}\right), z_{2}, \cdots, z_{n}\right)$ is $l-1$. Hence, from (19), there exists a normal full rank $(l-1) \times l$ submatrix $F_{1}\left(z_{2}, \cdots, z_{n}\right)$ $\triangleq F_{1}\left(f\left(z_{2}, \cdots, z_{n}\right), z_{2}, \cdots, z_{n}\right)$ of $F\left(f\left(z_{2}, \cdots, z_{n}\right), z_{2}, \cdots, z_{n}\right)$ such that

$$
F_{1}\left(z_{2}, \cdots, z_{n}\right)=A(z) \cdot \operatorname{diag}(0,1, \cdots, 1) \cdot V,
$$

where $A(z)$ is a $(l-1) \times l$ submatrix of $F_{0}\left(f\left(z_{2}, \cdots, z_{n}\right), z_{2}\right.$, $\left.\cdots, z_{n}\right) \cdot \overline{G_{0}}(z)$. By Lemma 3.5, the reduced minors of $F_{1}\left(z_{2}\right.$, $\left.\cdots, z_{n}\right)$ generate the unit ideal $K[z]$.

Sufficieny. Assuming that there exists a normal full rank $(l-1) \times l$ submatrix $F_{1}\left(z_{2}, \cdots, z_{n}\right) \triangleq F_{1}\left(f\left(z_{2}, \cdots, z_{n}\right), z_{2}, \cdots\right.$, $\left.z_{n}\right)$ of $F\left(f\left(z_{2}, \cdots, z_{n}\right), z_{2}, \cdots, z_{n}\right)$ such that the reduced minors of $F_{1}\left(z_{2}, \cdots, z_{n}\right)$ generate the unit ideal $K[z]$. According to Lemma 3.1, a ZRP vector $\omega \in K^{l \times 1}\left[z_{2}, \cdots, z_{n}\right]$ can be constructed such that

$$
F_{1}\left(z_{2}, \cdots, z_{n}\right) \cdot \omega\left(z_{2}, \cdots, z_{n}\right)=[0, \cdots, 0]^{T} .
$$

Since $F\left(f\left(z_{2}, \cdots, z_{n}\right), z_{2}, \cdots, z_{n}\right)$ is of normal rank $l-1$, by (21) and the fact that $F_{1}\left(z_{2}, \cdots, z_{n}\right)$ is a normal full rank submatrix of $F\left(f\left(z_{2}, \cdots, z_{n}\right), z_{2}, \cdots, z_{n}\right)$, we have

$$
F\left(f\left(z_{2}, \cdots, z_{n}\right), z_{2}, \cdots, z_{n}\right) \cdot \omega\left(z_{2}, \cdots, z_{n}\right)=[0, \cdots, 0]^{T}
$$

By Quillen-Suslin Theorem [26], a unimodular square matrix $V \in K^{l \times l}\left[z_{2}, \cdots, z_{n}\right]$ can be constructed such that $\omega$ $\left(z_{2}, \cdots, z_{n}\right)$ is its first column; combined with Lemma 2.1, we have

$F(z) \cdot V\left(z_{2}, \cdots, z_{n}\right)=F_{0}(z) \cdot \operatorname{diag}\left\{z_{1}-f\left(z_{2}, \cdots, z_{n}\right), 1, \cdots, 1\right\}$,

where $F_{0}(z) \in K^{m \times l}[z]$. It follows that

$$
F(z)=F_{0}(z) G_{0}(z)
$$

where $G_{0}(z)=\operatorname{diag}\left\{z_{1}-f\left(z_{2}, \cdots, z_{n}\right), 1, \cdots, 1\right\} \cdot V^{-1} \in K^{l \times l}$ $[z]$ with $\operatorname{det} G_{0}(z)=\left(z_{1}-f\left(z_{2}, \cdots, z_{n}\right)\right)=d(F)$.

Remark 3.1. When $K=C$ is the complex number field, according to Hilbert Zero Theorem, we see that the condition in Theorem 3.1 is the same as that of Theorem 2.1, and Theorem 3.1 is a generalization of Theorem 2.1. 
According to Theorem 3.1, in order to check that $F(z)$ satisfies the conditions of Theorem 3.1, we first find all the $(l-1) \times l$ submatrices of $F\left(f\left(z_{2}, \cdots, z_{n}\right), z_{2}, \cdots, z_{n}\right)$, denoted by $F_{1}, F_{2}, \ldots, F_{s}$, where $s=C_{m}^{l-1}=m(m-1) \cdots$ $(m-l) /(l-1) !$. For every $F_{i}(1 \leq i \leq s)$, we compute the $(l-1) \times(l-1)$ minors of $F_{i}$, saying as $a_{i 1}, a_{i 2}, \ldots, a_{i l}$. Let the ideal $I_{i}=\left\langle a_{i 1}, a_{i 2}, \cdots, a_{i l}\right\rangle$, we compute the reduced Gröbner basis $G_{i}$ of $I_{i}$. If $G_{i}$ contains only a polynomial $d_{i}(z)$, by the following result (Theorem 3.2), the reduced minors of $F_{i}$ generate the unit ideal $K[z]$.

Theorem 3.2. Let $d_{i}(z)$ be the greatest common divisor of $a_{i 1}, a_{i 2}, \ldots, a_{i l}$, i.e.,

$$
a_{i j}=b_{j} d_{i}(z), j=1,2, \ldots, l
$$

and $G_{i}$ be the reduced Gröbner basis of the ideal $I_{i}=\left\langle a_{i 1}\right.$, $\left.a_{i 2}, \cdots, a_{i l}\right\rangle$. Then $G_{i}$ contains only a polynomial $c_{i}(z)$ if and only if $b_{1}, b_{2}, \ldots, b_{l}$ generate the unit $K[z]$.

Proof 8. Necessity. Assuming that $G_{i}=\left\{c_{i}(z)\right\}$, it is obvious that $c_{i}(z) \mid a_{i j}$, where $j=1,2, \ldots, l$. Then $c_{i}(z) \mid d_{i}(z)$. Setting $d_{i}(z)=p_{i}(z) \cdot c_{i}(z)$, since $c_{i}(z) \in I_{i}$, we have that there are $u_{1}, u_{2}, \ldots, u_{l}$ such that

$$
\begin{aligned}
c_{i}(z)= & u_{1} a_{i 1}+u_{2} a_{i 2}+\cdots+u_{l} a_{i l}=u_{1} b_{1} d_{i}(z)+u_{2} b_{2} d_{i}(z) \\
& +\cdots+u_{l} b_{l} d_{i}(z) \\
d_{i}(z)= & p_{i}(z) c_{i}(z)=p_{i} u_{1} b_{1} d_{i}(z)+p_{i} u_{2} b_{2} d_{i}(z)+\cdots \\
& +p_{i} u_{l} b_{l} d_{i}(z)
\end{aligned}
$$

So $1=p_{i} u_{1} b_{1}+p_{i} u_{2} b_{2}+\cdots+p_{i} u_{l} b_{l}$. Thus, $b_{1}, b_{2}, \ldots, b_{l}$ generate the unit ideal $K[z]$.

Sufficiency. Assuming that $b_{1}, b_{2}, \ldots, b_{l}$ generate the unit ideal $K[z]$, then the reduced Gröbner basis of the ideal $\left\langle b_{1}, b_{2}, \cdots, b_{l}\right\rangle$ is $\{1\}$. Furthermore, the reduced Gröbner basis $G_{i}$ of $I_{i}=\left\langle b_{1} d_{i}(z), b_{2} d_{i}(z), \cdots, b_{l} d_{i}(z)\right\rangle$ is $\left\{d_{i}(z)\right\}$.

The reduced Gröbner basis algorithm of an ideal can be found in [16].

Remark 3.2. From the discussion above, we see that judging whether $F(z)$ satisfies the conditions of Theorem 3.1 or not, we only need to compute the reduced Gröbner basis of $I_{i}$, where $i=1,2, \ldots, l$.

Lin et al. studied the matrix factorization for a class of matrix whose g.c.d. of maximal order minors has divisor $\prod_{k=1}^{p}\left(z_{1}-z_{1 k}\right)$ ([13]); in the following, we study the minor prime factorization for this class of matrices and propose a tractable criterion.

Theorem 3.3. Let $K$ be an arbitrary field, $F(z) \in K^{m \times l}(l \leq m)$ be of normal full rank, $d(F)=\prod_{k=1}^{p}\left(z_{1}-z_{1 k}\right)$, where $z_{11}$, $z_{12}, \ldots, z_{1 p} \in K$ are distinguished. Then $F(z)$ admits a minor prime factorization $F(z)=F_{0}(z) G_{0}(z)$ with $F_{0}(z) \in K^{m \times l}[z]$,
$G_{0}(z) \in K^{l \times l}[z]$, and $\operatorname{det} G_{0}(z)=d(F)$ if and only if there are normal full rank $(l-1) \times l$ submatrices $F_{k}\left(z_{2}, \cdots, z_{n}\right)$ $\triangleq F_{k}\left(z_{1 k}, z_{2}, \cdots, z_{n}\right)$ of $F\left(z_{1 k}, z_{2}, \cdots, z_{n}\right)$ such that the reduced minors of $F_{k}\left(z_{2}, \cdots, z_{n}\right)$ generate the unit ideal $K[z]$, where $k=1,2, \ldots, p$.

Proof 9. Necessity. Supposing that $F(z)$ admits a minor prime factorization as follows:

$$
F(z)=F_{0}(z) G_{0}(z)
$$

where $F_{0}(z) \in K^{m \times l}[z], G_{0}(z) \in K^{l \times l}[z]$, and det $G_{0}(z)=d(F)$. For arbitrary but fixed $k(1 \leq k \leq p)$, from (27) and Lemma 3.4, we have

$$
\begin{aligned}
F\left(z_{1 k}, z_{2}, \cdots, z_{n}\right)= & F_{0}\left(z_{1 k}, z_{2}, \cdots, z_{n}\right) \cdot G_{0}\left(z_{1 k}, z_{2}, \cdots, z_{n}\right) \\
= & F_{0}\left(z_{1 k}, z_{2}, \cdots, z_{n}\right) \cdot \bar{G}\left(z_{1 k}, z_{2}, \cdots, z_{n}\right) \\
& \cdot \operatorname{diag}\{0,1, \cdots, 1\} \cdot V .
\end{aligned}
$$

By Lemma 3.2, we obtain that rank $F\left(z_{1 k}, z_{2}, \cdots, z_{n}\right)$ is $l-1$. Hence, from (28), there is a normal full rank $(l-1) \times l$ submatrix $F_{k}\left(z_{2}, \cdots, z_{n}\right) \triangleq F_{k}\left(z_{1 k}, z_{2}, \cdots, z_{n}\right)$ of $F\left(z_{1 k}, z_{2}\right.$, $\left.\cdots, z_{n}\right)$ such that

$$
F_{k}\left(z_{2}, \cdots, z_{n}\right)=A(z) \cdot \operatorname{diag}\{0,1, \cdots, 1\} \cdot V,
$$

where $A(z)$ is an $(l-1) \times l$ submatrix of $F_{0}\left(z_{1 k}, z_{2}, \cdots\right.$, $\left.z_{n}\right) \cdot \bar{G}\left(z_{1 k}, z_{2}, \cdots, z_{n}\right)$. By Lemma 3.5 , the reduced minors of $F_{k}\left(z_{2}, \cdots, z_{n}\right)$ also generate the unit ideal $K[z]$.

Sufficiency. If there exists a normal full rank $(l-1) \times l$ submatrix $F_{1}\left(z_{2}, \cdots, z_{n}\right) \triangleq F_{1}\left(z_{11}, z_{2}, \cdots, z_{n}\right)$ of $F\left(z_{11}, z_{2}, \cdots\right.$, $\left.z_{n}\right)$ such that the reduced minors of $F_{1}\left(z_{2}, \cdots, z_{n}\right)$ generate the unit ideal $K[z]$, according to Lemma 3.1, we can construct a ZRP vector $w_{1} \in K^{l \times l}\left[z_{2}, \cdots, z_{n}\right]$ such that

$$
F_{1}\left(z_{2}, \cdots, z_{n}\right) \cdot w_{1}\left(z_{2}, \cdots, z_{n}\right)=[0, \cdots, 0]^{T}
$$

By Lemma 3.2, $F\left(z_{11}, z_{2}, \cdots, z_{n}\right)$ is of normal rank $l-1$, from (30), and the fact that $F_{1}\left(z_{2}, \cdots, z_{n}\right)$ is a normal full rank submatrix of $F\left(z_{11}, z_{2}, \cdots, z_{n}\right)$, we have

$$
F\left(z_{11}, z_{2}, \cdots, z_{n}\right) \cdot w_{1}\left(z_{2}, \cdots, z_{n}\right)=[0, \cdots, 0]^{T} .
$$

By Quillen-Suslin Theorem [26], a unimodular square matrix $V_{1} \in K^{l \times l}\left[z_{2}, \cdots, z_{n}\right]$ can be constructed such that $w_{1}$ is its first columns; combined with Lemma 2.1, we have

$$
F(z) \cdot V_{1}\left(z_{2}, \cdots, z_{n}\right)=A_{1}(z) \cdot \operatorname{diag}\left(z_{1}-z_{11}, 1, \cdots, 1\right),
$$

where $A_{1}(z) \in K^{m \times l}[z]$. It follows that

$$
F(z)=A_{1}(z) \cdot G_{1}(z)
$$

where $G_{1}(z)=\operatorname{diag}\left(z_{1}-z_{11}, 1, \cdots, 1\right) \cdot V_{1}^{-1}$. 
It is obvious that $\operatorname{det} G_{1}(z)=z_{1}-z_{11}, d\left(A_{1}\right)=$ $\prod_{k=2}^{p}\left(z_{1}-z_{1 k}\right)$, and from (33), we have

$$
\begin{aligned}
F\left(z_{12}, z_{2}, \cdots, z_{n}\right)= & A_{1}\left(z_{12}, z_{2}, \cdots, z_{n}\right) \\
& \cdot \operatorname{diag}\left\{z_{12}-z_{11}, 1, \cdots, 1\right\} \cdot V_{1}^{-1}, \\
F_{2}\left(z_{12}, z_{2}, \cdots, z_{n}\right)= & A_{12}\left(z_{12}, z_{2}, \cdots, z_{n}\right) \\
& \cdot \operatorname{diag}\left\{z_{12}-z_{11}, 1, \cdots, 1\right\} \cdot V_{1}^{-1},
\end{aligned}
$$

where $A_{12}\left(z_{12}, z_{2}, \cdots, z_{n}\right)$ is an $(l-1) \times l$ submatrix of $A_{1}$ $\left(z_{12}, z_{2}, \cdots, z_{n}\right)$. By Lemma 3.2, rank $F\left(z_{12}, z_{2}, \cdots, z_{n}\right)$ is $l-1$ and from (34), rank $A_{1}\left(z_{12}, z_{2}, \cdots, z_{n}\right)$ is also $l-1$. By the assumption, the reduced minors of $F_{2}\left(z_{12}, z_{2}, \cdots, z_{n}\right)$ generate the unit ideal $K[z]$. Since $G_{1}\left(z_{12}, 1, \cdots, 1\right)=\operatorname{diag}\left\{z_{12}-z_{11}\right.$, $1, \cdots, 1\} \cdot V_{1}^{-1}$ is unimodular, by (35) and Cauchy-Binet formula, we obtain that the reduced minors of $A_{12}\left(z_{12}, z_{2}\right.$, $\left.\cdots, z_{n}\right)$ also generate the unit ideal $K[z]$.

Repeating the procedure above for $A_{1}(z)$ with respect to $\left(z_{1}-z_{12}\right)$, we have

$$
A_{1}(z)=A_{2}(z) \cdot G_{2}(z)
$$

or

$$
F(z)=A_{2}(z) \cdot G_{2}(z) \cdot G_{1}(z)
$$

such that $A_{2}(z) \in K^{m \times l}[z], G_{2}(z)=\operatorname{diag}\left(z_{1}-z_{12}, 1, \cdots, 1\right) \cdot$ $V_{2}^{-1}$ with det $G_{2}(z)=z_{1}-z_{12}, V_{2}$ is a unimodular square matrix, $d\left(A_{2}\right)=\prod_{k=3}^{p}\left(z_{1}-z_{1 k}\right)$. And

$$
\begin{aligned}
F_{3}\left(z_{13}, z_{2}, \cdots, z_{n}\right)= & A_{23}\left(z_{13}, z_{2}, \cdots, z_{n}\right) \\
& \cdot \operatorname{diag}\left(z_{13}-z_{12}, 1, \cdots, 1\right) \cdot V_{2}^{-1} \\
& \cdot \operatorname{diag}\left(z_{13}-z_{11}, 1, \cdots, 1\right) \cdot V_{1}^{-1},
\end{aligned}
$$

where $A_{23}\left(z_{13}, z_{2}, \cdots, z_{n}\right)$ is an $(l-1) \times l$ submatrix of $A_{2}\left(z_{13}, z_{2}, \cdots, z_{n}\right)$.

Furthermore, since $F_{3}\left(z_{13}, z_{2}, \cdots, z_{n}\right)$ is of normal rank $l-1$ and the reduced minors of $F_{3}\left(z_{13}, z_{2}, \cdots, z_{n}\right)$ generate the unit ideal $K[z]$, by (38), then $A_{23}\left(z_{13}, z_{2}, \cdots, z_{n}\right)$ is of normal rank $l-1$, and the reduced minors of $A_{23}\left(z_{13}\right.$, $\left.z_{2}, \cdots, z_{n}\right)$ also generate the unit ideal $K[z]$.

Repeating the same procedure continuously with respect to $\left(z_{1}-z_{1 k}\right), k=3, \ldots, p$, we obtain that

$$
F(z)=A_{p}(z) G_{p}(z) \cdots G_{2}(z) G_{1}(z)
$$

or

$$
F(z)=F_{0}(z) G_{0}(z)
$$

where $F_{0}(z)=A_{p}(z) \in K^{m \times l}[z], \quad G_{0}(z)=\prod_{k=1}^{p} G_{p}(z)$, det $G_{0}$ $(z)=\prod_{k=1}^{p}\left(z_{1}-z_{1 k}\right)$.

Remark 3.3. Similar to Remark 3.2, judging whether $F(z)$ satisfies the conditions of Theorem 3.1 or not, we only need to compute the reduced Gröbner bases of some ideals.

Example 3.1. Let $\mathbf{K} \triangleq \mathbf{Z} /(5)$ be the finite field, $F\left(z_{1}, z_{2}, z_{3}\right)$ $\in \mathbf{K}\left[z_{1}, z_{2}, z_{3}\right]$, and

$$
F\left(z_{1}, z_{2}, z_{3}\right)=\left(\begin{array}{cc}
2 z_{1}^{4}+4 z_{1}^{3} z_{2}^{2}+2 z_{1}^{3} z_{3}+4 z_{1} z_{3}+3 z_{2}^{2} z_{3}+4 z_{2}^{2}+4 z_{2} z_{3} & 3 z_{2}+4 z_{3} \\
3 z_{1}^{2}+z_{1} z_{2}^{2}+2 z_{1} z_{2}^{2}+4 z_{1} z_{2}+4 z_{2}^{4}+2 z_{2}^{2} z_{3} & 3 z_{1} \\
4 z_{1}^{2} z_{2}+z_{1} z_{2}^{2} z_{3}+3 z_{1} z_{3}^{2}+2 z_{1} z_{3}+4 z_{2}^{2} z_{3}+z_{2} z_{3} & 3 z_{1}^{2}+2 z_{3}
\end{array}\right)
$$

It is easy to check that the greatest common divisor of the $2 \times 2$ minors of $F(z)$ is $d(F)=z_{1}+2 z_{2}^{2}+z_{3}=z_{1}$ $\left(3 z_{2}^{2}+4 z_{3}\right)$. By computing, we have

$$
F\left(3 z_{2}^{2}+4 z_{3}, z_{2}, z_{3}\right)=\left(\begin{array}{cc}
4 z_{2}^{2}+4 z_{2} z_{3}+z_{3}^{2} & 3 z_{2}+4 z_{3} \\
2 z_{2}^{3}+z_{2}^{2} z_{3}+z_{2} z_{3}+3 z_{3}^{2} & 4 z_{2}^{2}+2 z_{3} \\
4 z_{2}^{5}+3 z_{2}^{4} z_{3}+4 z_{2}^{3} z_{3}+3 z_{2}^{2} z_{2}^{3}+z_{2} z_{3}^{2}+z_{2} z_{3} & 2 z_{2}^{4}+2 z_{2}^{2} z_{3}+3 z_{3}^{3}+2 z_{3}
\end{array}\right)
$$

Obviously, $F\left(3 z_{2}^{2}+4 z_{3}, z_{2}, z_{3}\right)$ is of normal rank 1 , the reduced minors of the first row of $F\left(3 z_{2}^{2}+4 z_{3}, z_{2}, z_{3}\right)$ (a 1 $\times 2$ submatrix of $\left.F\left(3 z_{2}^{2}+4 z_{3}, z_{2}, z_{3}\right)\right)$ are $3 z_{2}+4 z_{3}$ and 1 (since $\left.4 z_{2}^{2}+4 z_{2} z_{3}+z_{3}^{2}=\left(3 z_{2}+4 z_{3}\right)\left(3 z_{2}+4 z_{3}\right)\right)$, which generate the unit ideal $\mathbf{K}[z]$. By Theorem 3.1, there is a minor prime factorization for $F\left(z_{1}, z_{2}, z_{3}\right)$. 
To construct such a factorization, let

$$
U=\left(\begin{array}{cc}
3 & 0 \\
z_{2}+3 z_{3} & 2
\end{array}\right)
$$

we have

$$
F\left(3 z_{2}^{2}+4 z_{3}, z_{2}, z_{3}\right) \cdot U=\left(\begin{array}{cc}
0 & z_{2}+3 z_{3} \\
0 & 3 z_{2}^{2}+4 z_{3} \\
0 & 4 z_{2}^{4}+4 z_{2}^{2} z_{3}+z_{3}^{2}+4 z_{3}
\end{array}\right)
$$

which gives

$$
\begin{aligned}
F\left(z_{1}, z_{2}, z_{3}\right) \cdot U= & \left(\begin{array}{cc}
z_{1}^{3}+2 z_{3} & z_{2}+3 z_{3} \\
4 z_{1}+z_{2}^{2} & z_{1} \\
4 z_{1} z_{3}+z_{3} & z_{1}^{2}+4 z_{3}
\end{array}\right) \\
& \cdot\left(\begin{array}{cc}
z_{1}+2 z_{2}^{2}+z_{3} & 0 \\
0 & 1
\end{array}\right) .
\end{aligned}
$$

Let

$$
\begin{aligned}
F_{0}(z)= & \left(\begin{array}{cc}
z_{1}^{3}+2 z_{3} & z_{2}+3 z_{3} \\
4 z_{1}+z_{2}^{2} & z_{1} \\
4 z_{1} z_{3}+z_{3} & z_{1}^{2}+4 z_{3}
\end{array}\right), \\
G_{0}(z)= & \left(\begin{array}{cc}
z_{1}+2 z_{2}^{2}+z_{3} & 0 \\
0 & 1
\end{array}\right) \\
& \cdot\left(\begin{array}{cc}
2 & 0 \\
4 z_{2}+2 z_{3} & 3
\end{array}\right)
\end{aligned}
$$

we have

$$
F\left(z_{1}, z_{2}, z_{3}\right)=F_{0}\left(z_{1}, z_{2}, z_{3}\right) \cdot G_{0}\left(z_{1}, z_{2}, z_{3}\right) .
$$

Remark 3.4. In our two main theorems (Theorem 3.1 and 3.3), $F(z) \in K^{m \times l}(l \leq m)$ are of normal full rank. In fact, our methods can also deal with some matrices not of full normal rank. When the normal rank of $F(z)$ is $r$ and $r<l$, if the reduce minors of $F(z)$ generate the unit ideal $K[z]$, one has that $F(z)=Q_{1}(z) B_{1}(z)$ such that $Q_{1} \in K^{m \times r}$ of normal full rank, and $B_{1} \in K^{r \times l}$ is ZRP. In order to consider the factorization of $F(z)$, one needs to consider the factorization of $Q_{1}(z)$. See the following example.

Example 3.2. Let $K$ be the rational number field, $A(z) \in$ $K^{3 \times 3}\left[z_{1}, z_{2}, z_{3}\right]$, and

$$
A(z)=\left(\begin{array}{ccc}
3 z_{1}^{2}-4 z_{1} z_{2}-4 z_{2} & 3 z_{1}^{2} z_{2}-4 z_{1}^{2} z_{2}+z_{1} z_{2}-1 & -3 z_{1}^{2} z_{2}+4 z_{1} z_{2}-z_{2} \\
0 & z_{1}-3 & 0 \\
z_{1}^{2} z_{2} z_{3}-z_{1} z_{2} z_{3} & z_{1}^{3} z_{2} z_{3}-z_{1}^{2} z_{2} z_{3}+3 z_{3} & -z_{1}^{2} z_{2} z_{3}+2 z_{1} z_{3}
\end{array}\right) .
$$

By computing the minors of $A(z)$, we have that rank $A(z)=2, d(A)=\left(z_{1}-1\right)\left(z_{1}-3\right)$, the reduced minors of $A$ $(z)$ generate the unit ideal $K[z]$. Using Theorem 3.2 in [18], we have that $A(z)=F(z) B(z)$, where

$$
\begin{aligned}
& F(z)=\left(\begin{array}{cc}
4 z_{1} z_{2}-4 z_{2} & z_{1}-1 \\
3 z_{1} z_{2}-9 z_{2} & z_{1}-3 \\
-z_{1}^{2} z_{2} z_{3}+z_{1} z_{2} z_{3} & 3 z_{3}
\end{array}\right), \\
& B(z)=\left(\begin{array}{ccc}
-1 & z_{1} & 1 \\
3 z_{2} & 1+3 z_{1} z_{2} & z-3 z_{2}
\end{array}\right),
\end{aligned}
$$

and $B(z)$ is ZLP. It is easy to check that the greatest common divisor of the $2 \times 2$ minors of $F(z)$ is $d(F)=z_{1}^{2}-4$ $z_{1}+3=\left(z_{1}-1\right)\left(z_{1}-3\right)$. Obviously, $F\left(1, z_{2}, z_{3}\right)$ is of normal rank 1 , the reduced minors of the second row of $F\left(1, z_{2}, z_{3}\right) \quad\left(\right.$ a $1 \times 2$ submatrix of $\left.F\left(1, z_{2}, z_{3}\right)\right)$ are $-6 z_{2}$ and -2 , which generate the unit ideal $K[z] . F\left(3, z_{2}, z_{3}\right)$ is also of normal rank 1 , the reduced minors of the first row of $F\left(3, z_{2}, z_{3}\right)$ are $8 z_{2}$ and 8 , which also generate the unit ideal $K[z]$. By Theorem 3.3, there is a minor prime factorization for $F\left(z_{1}, z_{2}, z_{3}\right)$.

To construct such a factorization, let

$$
U_{1}=\left(\begin{array}{cc}
1 & 0 \\
-3 z_{2} & 1
\end{array}\right)
$$

we have

$$
F\left(1, z_{2}, z_{3}\right)=\left(\begin{array}{cc}
0 & 0 \\
-6 z_{2} & -2 \\
9 z_{2} z_{3} & 3 z_{3}
\end{array}\right) \text {, }
$$




$$
F\left(1, z_{2}, z_{3}\right) \cdot U_{1}=\left(\begin{array}{cc}
0 & 0 \\
0 & -2 \\
0 & 0
\end{array}\right)
$$

which gives

$F\left(z_{1}, z_{2}, z_{3}\right) \cdot U_{1}=\left(\begin{array}{cc}-3 z_{1} z_{2}+z_{2} & z_{1}^{2}-1 \\ 0 & z_{1}-3 \\ -z_{1} z_{2} z_{3} & 3 z_{3}\end{array}\right) \cdot\left(\begin{array}{cc}z_{1}-1 & 0 \\ 0 & 1\end{array}\right)$.

Thus,

$$
F\left(z_{1}, z_{2}, z_{3}\right)=\left(\begin{array}{cc}
-3 z_{1} z_{2}+z_{2} & z_{1}^{2}-1 \\
0 & z_{1}-3 \\
-z_{1} z_{2} z_{3} & 3 z_{3}
\end{array}\right) \cdot\left(\begin{array}{cc}
z_{1}-1 & 0 \\
3 z_{2} & 1
\end{array}\right) .
$$

Let

$$
\begin{aligned}
F_{1}\left(z_{1}, z_{2}, z_{3}\right) & =\left(\begin{array}{cc}
-3 z_{1} z_{2}+z_{2} & z_{1}^{2}-1 \\
0 & z_{1}-3 \\
-z_{1} z_{2} z_{3} & 3 z_{3}
\end{array}\right), \\
U_{2} & =\left(\begin{array}{cc}
1 & 0 \\
z_{2} & 1
\end{array}\right),
\end{aligned}
$$

we have

$$
\begin{aligned}
F_{1}\left(3, z_{2}, z_{3}\right) & =\left(\begin{array}{cc}
-8 z_{2} & 8 \\
0 & 0 \\
-3 z_{2} z_{3} & 3 z_{3}
\end{array}\right), \\
F_{1}\left(3, z_{2}, z_{3}\right) \cdot U_{2} & =\left(\begin{array}{cc}
0 & 8 \\
0 & 0 \\
0 & 3 z_{3}
\end{array}\right),
\end{aligned}
$$

which gives

$$
F_{1}\left(z_{1}, z_{2}, z_{3}\right) \cdot U_{2}=\left(\begin{array}{cc}
z_{1} z_{2} & z_{1}^{2}-1 \\
z_{2} & z_{1}-3 \\
-z_{2} z_{3} & 3 z_{3}
\end{array}\right) \cdot\left(\begin{array}{cc}
z_{1}-3 & 0 \\
0 & 1
\end{array}\right)
$$

Thus,

$$
\begin{aligned}
F_{1}\left(z_{1}, z_{2}, z_{3}\right)= & \left(\begin{array}{cc}
z_{1} z_{2} & z_{1}^{2}-1 \\
z_{2} & z_{1}-3 \\
-z_{2} z_{3} & 3 z_{3}
\end{array}\right) \cdot\left(\begin{array}{cc}
z_{1}-3 & 0 \\
-z_{2} & 1
\end{array}\right), \\
F\left(z_{1}, z_{2}, z_{3}\right)= & \left(\begin{array}{cc}
z_{1} z_{2} & z_{1}^{2}-1 \\
z_{2} & z_{1}-3 \\
-z_{2} z_{3} & 3 z_{3}
\end{array}\right) \cdot\left(\begin{array}{cc}
z_{1}-3 & 0 \\
-z_{2} & 1
\end{array}\right) \\
& \cdot\left(\begin{array}{cc}
z_{1}-1 & 0 \\
3 z_{2} & 1
\end{array}\right) .
\end{aligned}
$$

Let

$$
\begin{aligned}
F_{0}(z) & =\left(\begin{array}{cc}
z_{1} z_{2} & z_{1}^{2}-1 \\
z_{2} & z_{1}-3 \\
-z_{2} z_{3} & 3 z_{3}
\end{array}\right), \\
G_{0}(z) & =\left(\begin{array}{cc}
z_{1}-3 & 0 \\
-z_{2} & 1
\end{array}\right) \cdot\left(\begin{array}{cc}
z_{1}-1 & 0 \\
3 z_{2} & 1
\end{array}\right),
\end{aligned}
$$

it is clear that det $G_{0}(z)=z_{1}^{2}-4 z_{1}+3=d(A)$. We have

$$
\begin{aligned}
& F\left(z_{1}, z_{2}, z_{3}\right)=F_{0}\left(z_{1}, z_{2}, z_{3}\right) \cdot G_{0}\left(z_{1}, z_{2}, z_{3}\right), \\
& A\left(z_{1}, z_{2}, z_{3}\right)=F_{0}\left(z_{1}, z_{2}, z_{3}\right) \cdot G_{0}\left(z_{1}, z_{2}, z_{3}\right) \cdot B\left(z_{1}, z_{2}, z_{3}\right),
\end{aligned}
$$

where $B\left(z_{1}, z_{2}, z_{3}\right)$ is ZLP.

The desired minor prime factorization for $A\left(z_{1}, z_{2}, z_{3}\right)$ is obtained.

\section{Conclusions}

In this paper, we have investigated the minor prime factorization for two classes of $n$-D polynomial matrices whose g.c.d. of maximal order minors is $z_{1}-f\left(z_{2}, \cdots, z_{n}\right)$ or $\prod_{k=1}^{p}$ $\left(z_{1}-z_{1 k}\right)$ with their entries in $K[z]$, where $K$ is an arbitrary field. We have presented some key lemmas and two tractable criterions for the existence of minor prime factorizations. These criterions are easily checked by computing reduced Gröbner basis of the ideal generated by lower order minors, and the minor prime factorization can be carried out by Lin's constructive methods.

\section{Data Availability}

No data were used to support this study.

\section{Conflicts of Interest}

The authors declare that they have no conflicts of interest. 


\section{Acknowledgments}

The authors are grateful to the anonymous referees for instructive and helpful comments on this paper. This research is supported by the National Science Foundation of China (11871207, 11501192 and 11471108) and the Scientific Research Fund of Hunan Province Education Department (2017JJ3084).

\section{References}

[1] H. H. Rosenbrock, State Space and Multivariable Theory, Nelson-Wiley, London, New York, 1970.

[2] N. Bose, Applied Multidimensional Systems Theory, Van Nostrand Reinhold, New York, 1982.

[3] N. Bose, B. Buchberger, and J. Guiver, Multidimensional Systems Theory and Applications, Kluwer, Dordrecht, The Netherlands, 2003.

[4] E. Fornasini and M. E. Valcher, " $n \mathrm{D}$ polynomial matrices with applications to multidimensional signal analysis," Multidimensional Systems and Signal Processing, vol. 8, no. 4, pp. 387-408, 1997.

[5] Z. Lin, L. Xu, and N. K. Bose, "A tutorial on Gröbner bases with applications in signals and systems," IEEE Transactions on Circuits and Systems I: Regular Papers, vol. 55, no. 1, pp. 445-461, 2008.

[6] T. Kailath, Linear Systems, Prentice Hall, Englewood Cliffs, NJ, USA, 1980.

[7] M. S. Boudellioua and A. Quadrat, "Serre's reduction of linear functional systems," Mathematics in Computer Science, vol. 4, no. 2-3, pp. 289-312, 2010.

[8] D. Youla and G. Gnavi, "Notes on $n$-dimensional system theory," IEEE Transactions on Circuits and Systems, vol. 26, no. 2, pp. 105-111, 1979.

[9] J. Guiver and N. Bose, "Polynomial matrix primitive factorization over arbitrary coefficient field and related results," IEEE Transactions on Circuits and Systems, vol. 29, no. 10, pp. 649-657, 1982.

[10] Z. Lin, "On primitive factorization for $n$-D polynomial matrices," in 1993 IEEE International Symposium on Circuits and Systems, pp. 595-598, Chicago, IL, USA, May 1993.

[11] M. G. Frost and M. S. Boudellioua, "Some further results concerning matrices with elements in a polynomial ring," International Journal of Control, vol. 43, no. 5, pp. 15431555, 1986.

[12] Z. Lin, "Notes on $n$-D polynomial matrix factorization," Multidimensional Systems and Signal Processing, vol. 10, no. 4, pp. 379-393, 1999.

[13] Z. Lin, J. Q. Ying, and L. Xu, "Factorizations for $n \mathrm{D}$ polynomial matrices," Circuits, Systems and Signal Processing, vol. 20, no. 6, pp. 601-618, 2001.

[14] Z. Lin, L. Xu, and H. Fan, "On minor prime factorizations for $n$ -D polynomial matrices," IEEE Transactions on Circuits and Systems II:Express Briefs, vol. 52, no. 9, pp. 568-571, 2005.

[15] Z. Lin and N. K. Bose, "A generalization of Serre's conjecture and some related issues," Linear Algebra and its Applications, vol. 338, no. 1-3, pp. 125-138, 2001.

[16] G.-M. Greuel and G. Pfister, A Singular Introduction to Commutative Algebra, Spinger-Verlag, New York, 2002.
[17] J. F. Pommaret, "Solving Bose conjecture on linear multidimensional systems," in 2001 European Control Conference (ECC), pp. 1853-1855, Porto, Portugal, September 2001.

[18] M. Wang and D. Feng, "On Lin-Bose problem," Linear Algebra and its Applications, vol. 390, pp. 279-285, 2004.

[19] V. Srinivas, “A generalized Serre problem," Journal of Algebra, vol. 278, no. 2, pp. 621-627, 2004.

[20] J. Liu, D. Li, and L. Zheng, "The Lin-Bose problem," IEEE Transactions on Circuits and Systems II: Express Briefs, vol. 61, no. 1, pp. 41-43, 2014.

[21] Z. Lin, "On matrix fraction descriptions of multivariable linear $n$-D systems," IEEE Transactions on Circuits and Systems, vol. 35, no. 10, pp. 1317-1322, 1988.

[22] W. Mingsheng and C. P. Kwong, "On multivariate polynomial matrix factorization problems," Mathematics of Control, Signals, and Systems, vol. 17, no. 4, pp. 297-311, 2005.

[23] M. Wang, "On factor prime factorization for $n$-D polynomial matrices," IEEE Transactions on Circuits and Systems I: Regular Papers, vol. 54, no. 6, pp. 1398-1405, 2007.

[24] M. Wang, "Remarks on $n$-D polynomial matrix factorization problems," IEEE Transactions on Circuits and Systems II: Express Briefs, vol. 55, no. 1, pp. 61-64, 2008.

[25] J. Liu and M. Wang, "Notes on factor prime factorizations for $n$-D polynomial matrices," Multidimensional Systems and Signal Processing, vol. 21, no. 1, pp. 87-97, 2010.

[26] D. Quillen, "Projective modules over polynomial rings," Inventiones Mathematicae, vol. 36, no. 1, pp. 167-171, 1976. 


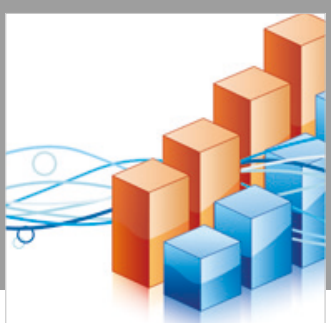

Advances in

Operations Research

\section{-n-m}
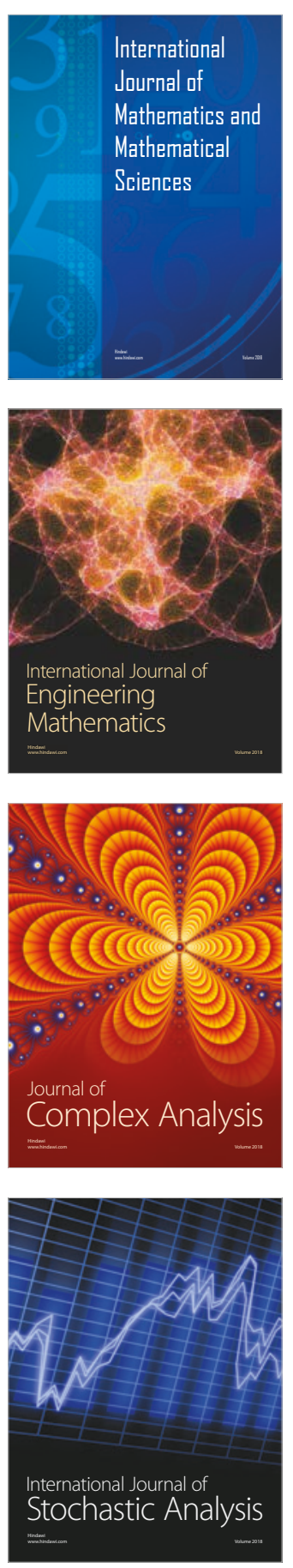
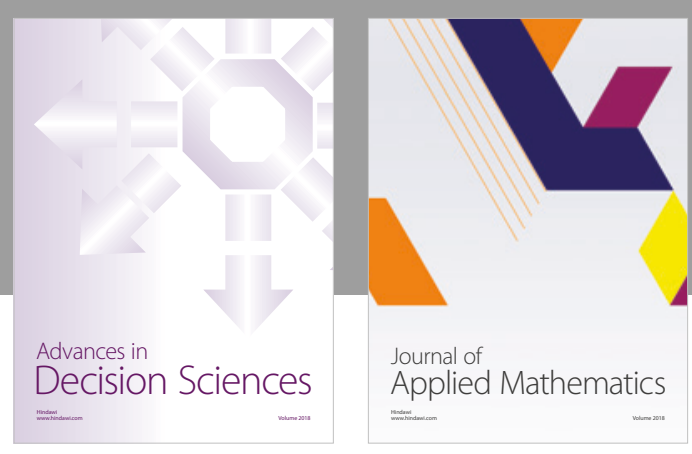

Journal of

Applied Mathematics
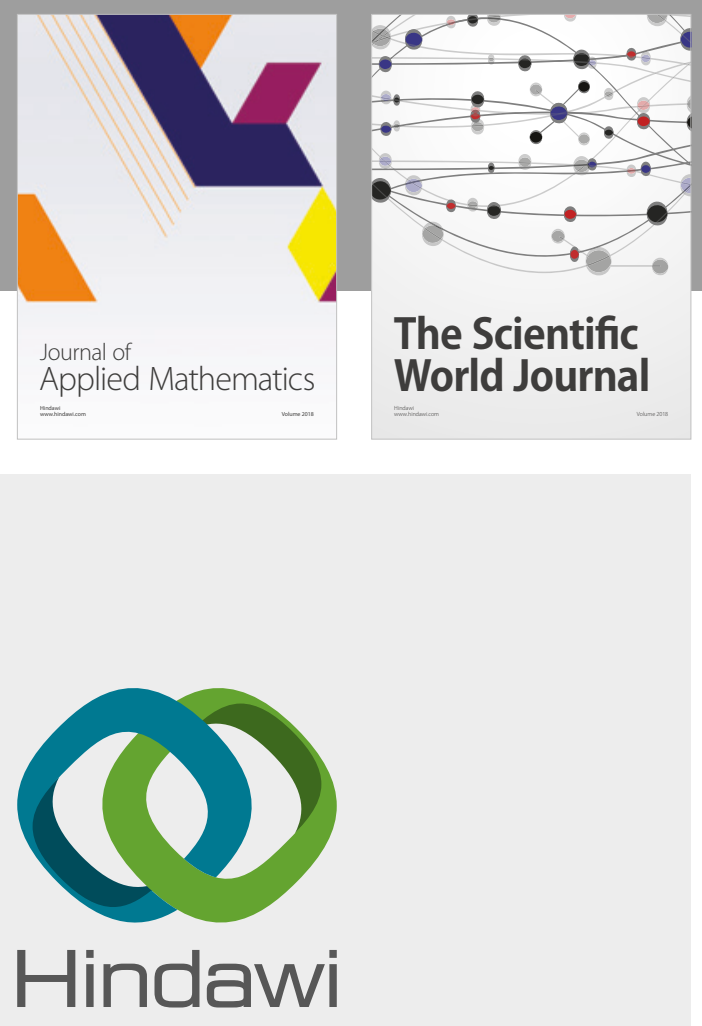

Submit your manuscripts at

www.hindawi.com

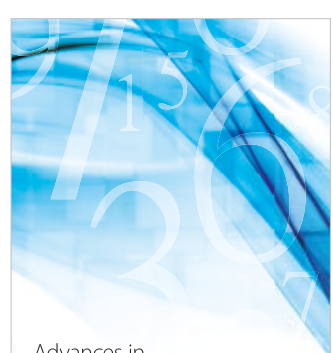

Advances in
Numerical Analysis
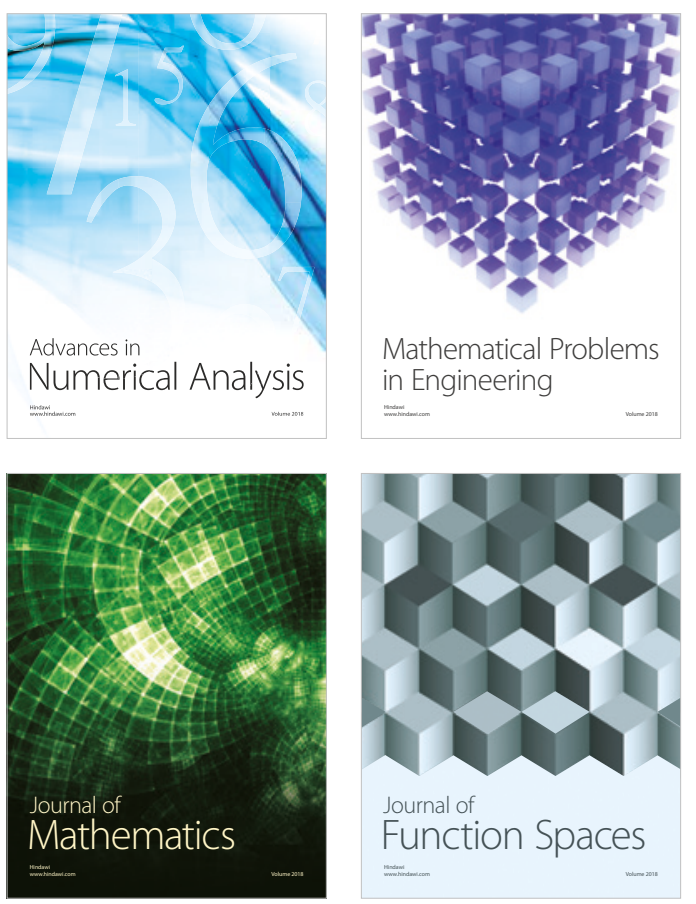

Mathematical Problems in Engineering

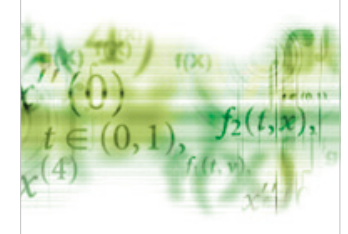

International Journal of

Differential Equations

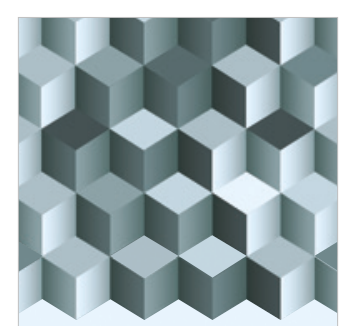

Journal of

Function Spaces
The Scientific

World Journal

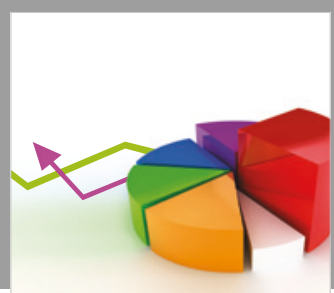

Journal of

Probability and Statistics
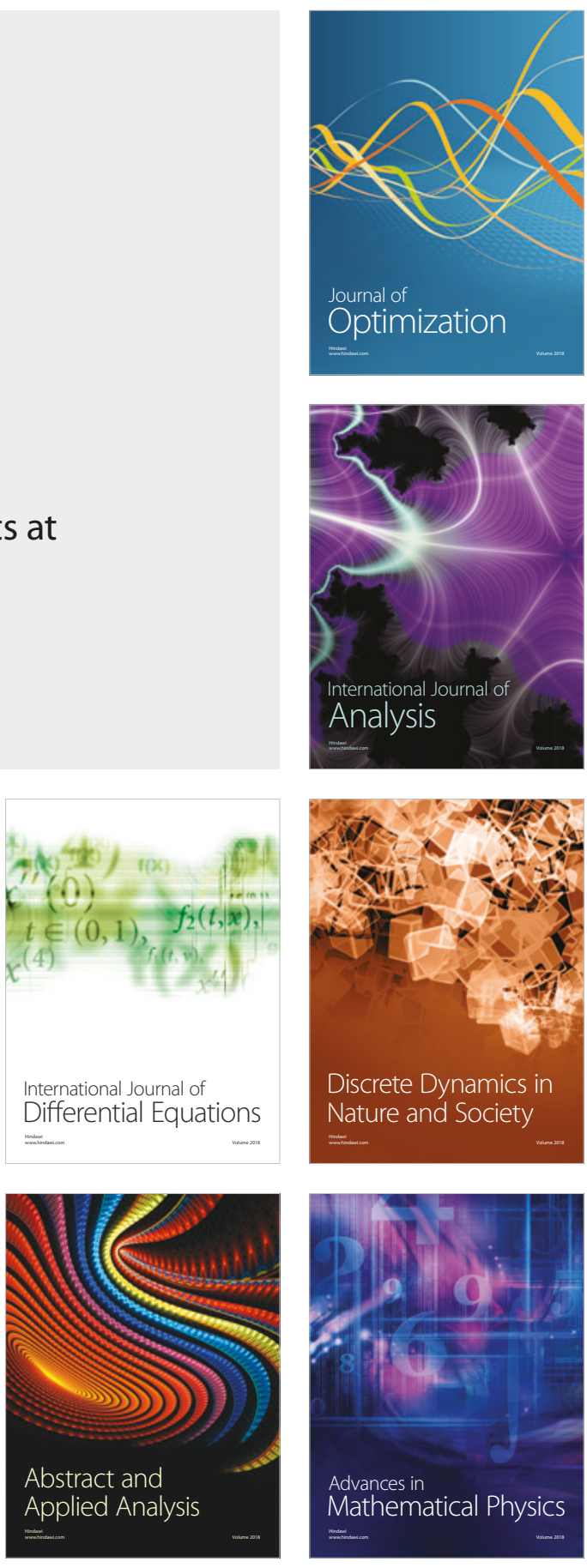